\title{
Mapa Situacional do Marketing: Um condensado das bibliometrias publicadas na área
}

\author{
Situational Marketing Map: A condensate of published bibliometries in the area \\ Gilmara Aparecida de Freitas Dias \\ Karla Veloso Coura ${ }^{2}$ \\ André Luiz Mendes Athayde ${ }^{3}$
}

\begin{abstract}
Resumo: Objetivos: A presente pesquisa objetivou analisar as bibliometrias da área de marketing, tendo como base de dados a Scientific Periodicals Electronic Library (Spell). Para tanto, buscou-se identificar elementos como temáticas, demografia, estatísticas textuais, especificidades de grupos, Classificação Hierárquica Descendente e Nuvens de Palavras no corpus textual construído com os dados dos artigos. Método: Este estudo se baseou na aplicação do método bibliométrico em 37 bibliometrias com conteúdo de marketing disponibilizadas na Spell. A análise dos artigos dividiu-se em dois blocos: o primeiro abordou os artigos da amostra e o segundo resumiu as informações dos artigos tratados no primeiro. Resultados: Os resultados retrataram que as temáticas 'consumo' e 'estratégia' foram as mais estudadas e os artigos foram escritos de forma conjunta em sua maioria. Os estudos foram majoritariamente teórico-empíricos e com abordagem qualitativa. Adicionalmente, a sociometria desenvolvida na presente pesquisa revelou a formação de três clusters, além de serem analisadas a Classificação Hierárquica Descendente (CHD) e a Análise Fatorial de Correspondência (AFC). Conclusões: caracterizou-se a área de estudo até então, embasando os pesquisadores para futuras investigações por meio de uma agenda de trabalho em pesquisa.
\end{abstract}

Palavras-chave: Marketing. Consumidor. Estudo de Bibliometrias. Produção Nacional.

Abstract: Objectives: This research aimed to analyze the bibliometries of the marketing area, using the Scientific Periodicals Electronic Library (Spell) as a database. To this end, we sought to identify elements such as themes, demography, textual statistics, group specificities, Descending Hierarchical Classification and Word Clouds in the textual corpus constructed with the data from the articles. Method: This study was based on the application of the bibliometric method in 37 bibliometries with marketing content available at Spell. The analysis of the

\footnotetext{
${ }^{1}$ Mestre em Administração pela Universidade Federal de Viçosa (UFV). Professor da Universidade Estadual de Montes Claros (UNIMONTES). Minas Gerais, Brasil. $₫$ gilfdias@ gmail.com.

${ }^{2}$ Doutora em Administração pela Universidade de Brasília (UNB). Professora da Universidade Estadual de Montes Claros (UNIMONTES). Minas Gerais, Brasil. $\triangle$ karlaveco@bol.com.br (Dhttps://orcid.org/0000-0002-73020403.

${ }^{3}$ Doutor em Administração pela Universidade de Brasília (UNB). Professor da Universidade Federal de Minas Gerais (UFMG), Campus Montes Claros. Minas Gerais, Brasil. $\$ andreluizathayde@outlook.com https://orcid.org/0000-0002-2109-2130

$\begin{array}{ccc}\text { Recebido em } & \text { Aceito em } & \text { Publicado em } \\ 25 / 03 / 2019 & 24 / 10 / 2019 & 14 / 12 / 2020\end{array}$
}


articles was divided into two blocks: the first approached the sample articles and the second summarized the information of the articles treated in the first. Results: The results showed that the themes 'consumption' and 'strategy' were the most studied and the articles were mostly written together. The studies were mostly theoretical-empirical and with a qualitative approach. Additionally, the sociometry developed in the present research revealed the formation of three clusters, in addition to analyzing the Descending Hierarchical Classification (CHD) and the Correspondence Factor Analysis (AFC). Conclusions: the study area was characterized until then, basing researchers for future investigations through a research work agenda.

Keywords: Marketing. Consumer. Study of Bibliometrics. National Production.

\section{INTRODUÇÃO}

Os primeiros estudos da área de marketing, em meados de 1880, são advindos da ciência econômica (BARTELS, 1988). Apesar disso, a aquisição de uma forma mais consistente foi adquirida apenas por volta do início de 1960, devido às novas condições econômicas e sociais, por meio das contribuições de variados autores como Joel Dean, Theodore Levitt e Eugene McCarthy (BAKER, 2000).

De acordo com Bartels (1988), os primeiros encontros da área de marketing ocorreram a partir de 1915. O autor cita a seção especial de marketing na American Economic Association (Associação Econômica Americana), em 1918, que resultou em 1920, na National Association of Teachers of Maketing and Advertising (Associação Nacional de Professores de Marketing e Publicidade) como um dos pontos para o avanço do campo. Em 1930, foi criada a American Marketing Society (Sociedade Americana de Marketing) e, hoje, uma das principais associações do marketing, criada desde 1937, é a American Marketing Association (Associação Americana de Marketing- AMA).

A partir desses acontecimentos e com os passar dos anos, o marketing assumiu variadas perspectivas no que diz respeito aos conceitos e papel nas organizações. Para Baker (2000), as definições do que vem a ser marketing variam de acordo com a natureza do objeto da pesquisa, suas aplicações, suas funções e seus resultados. No entanto, verifica-se que, mesmo com o desenvolvimento da teoria, o foco nos consumidores não deixou de existir, sendo eles fator primordial para a continuidade das organizações. Nessa ótica, a AMA (2017) conceitua o marketing como "uma atividade, conjunto de instituições e processos para criar, comunicar, entregar e trocar ofertas que tenham valor para clientes, parceiros e a sociedade em geral". Segundo a referida associação, a pesquisa de marketing tem como função a ligação entre consumidor, cliente e público com o comerciante por meio da informação. 
A cientificidade da disciplina de marketing, por sua vez, constitui um dos grandes debates na área (HUNT, 1983). Segundo Hunt (2002), após décadas de discussão sobre essa questão, a cientificidade do marketing pode contribuir para a construção genuína do conhecimento. Para Anderson (1983), o marketing pode ser considerado científico na medida em que, como disciplina, produz conhecimento que se torna relevante para a academia e sociedade.

A difusão do conhecimento, instrumentos de registro, desenvolvimento da tecnologia e da inovação devem ser a base para a gestão de pesquisas científicas (VANTI, 2002). Nessa verve, tem-se que a produção do conhecimento deve ter como objetivo a evolução da ciência. Assim, a bibliometria tem como objetivo a ordenação de indicadores, de temáticas, além de proporcionar lacunas para futuros estudos. Para Tellis, Chandy e Ackerman (1999), a preocupação com trabalhos deve englobar a descrição do que já foi publicado em comparação com o que deveria ser, quanto aos estudos da área de marketing.

Paul Otlet foi o criador do termo 'bibliometria' em 1930, uma ciência que, até então, era conhecida como 'bibliografia estatística' (HULME, 1923). A pesquisa bibliométrica tem como objetivo a mensuração e análise da produção científica (PRITCHARD, 1969). Complementando, esse autor enfatiza que a bibliometria denota "todos os estudos que tentam quantificar os processos de comunicação escrita" (PRITCHARD, 1969, p. 348). Guedes e Borschiver (2005) apresentam a bibliometria como uma ferramenta estatística que busca a geração de conhecimento e informação de uma área ou temática. Trata-se de uma ferramenta que busca mapear e gerar indicadores variados que podem auxiliar no planejamento e gestão da ciência e da tecnologia (KLEINUBING, 2010).

A bibliometria é regida por três leis: de Lotka, criada em 1926; de Brandford, em 1934; e de Zipf, em 1949. A primeira lei está associada ao relacionamento da produtividade dos autores, verificando os que produzem mais e os que produzem menos; a segunda tem como foco a produtividade dos periódicos, como exemplo o número de artigos sobre determinado assunto; já a lei de Zipf observa as frequências das palavras de determinado trabalho (GUEDES; BORSHIVER, 2005; ARAÚJO, 2006).

Nesse contexto, o trabalho dos autores Machado-da-Silva, Cunha e Amboni (1990) foi o primeiro trabalho brasileiro, publicado na década de 90 , que apresentou a abordagem de pesquisa através do método bibliométrico. O estudo foi embasado nos artigos do campo de organizações, entre os anos de 1985 e 1989, realizado em periódicos de Administração brasileiros. Posteriormente, acompanhando essa perspectiva, outras áreas, como gestão de 
pessoas, administração pública, finanças, estratégia e operações também introduziram essa metodologia.

Com relação à área de marketing, pode-se destacar os trabalhos bibliométricos nacionais de Vieira (1998, 1999, 2000, 2002, 2003); Perin et al. (2000); Brei e Liberali (2004) e Faria (2006); e os trabalhos bibliométricos internacionais de Leong (1989); Cote, Leong e Cote (1991); Kerin (1996); Bakir, Vitell e Rose (2000); Leone et al. (2011) e Talukdar, Hariharan e Boo (2011).

Com os avanços dessa metodologia, as categorias e variáveis apresentadas nos artigos bibliométricos têm sofrido alterações durante o tempo e, assim, estão surgindo diversas análises com a utilização de diferentes exposições de dados e aplicações de softwares. Dado esse cenário, a presente pesquisa propõe os seguintes questionamentos: Como está o estado das pesquisas sobre marketing publicadas nos periódicos de nível nacional? Quais os temas já foram trabalhados nas bibliometrias da área? O que está sendo utilizado para representação e análise dos dados? Este estudo, portanto, tem um caráter inovador ao elaborar uma bibliometria de bibliometrias.

Assim, focando em proporcionar de maneira condensada um panorama da área de marketing, este trabalho teve como objetivo precípuo analisar as bibliometrias da área de marketing, tendo como base de dados a Spell. Ademais, buscou-se identificar elementos como temáticas; demografia; estatísticas textuais; especificidades de grupos; Classificação Hierárquica Descendente e Nuvens de Palavras no corpus textual construído com os dados dos artigos, e como embasar os pesquisadores para futuros trabalhos na área por meio de uma agenda de pesquisa.

Para consecução dos objetivos propostos, será enunciada em primeira instância a metodologia adotada, englobando as palavras-chave, categorias e variáveis a serem apresentadas nos resultados e como a análise deste estudo será conduzida. Em seguida, serão descritos os resultados engendrados. A posteriori, uma agenda de pesquisa para futuros trabalhos será traçada a partir do levantamento realizado. Por fim, serão feitas as considerações finais.

\section{MÉTODO}

Visando à consecução dos objetivos deste estudo, adotou-se a abordagem bibliométrica para o recolhimento dos dados, uma técnica quantitativa e estatística que detecta modelos ou 
padrões de pesquisas e envolve o desenvolvimento e a disseminação do conhecimento científico (RAMOS-RODRÍGUEZ; RUÍZ-NAVARRO, 2004).

O presente estudo, de natureza exploratória, seguiu alguns critérios em relação à seleção da base de busca, à definição das categorias a serem analisadas e ao enquadramento dos artigos selecionados de acordo com tais categorias. A investigação sistemática dos artigos bibliométricos da área de marketing, como ressaltado, considerou a base de dados da Scientific Periodicals Electronic Library (Spell), que engloba os principais periódicos nacionais das áreas de Administração, Contabilidade e Turismo.

Após essa etapa, os artigos pesquisados devem conter os descritores (Figura 1) nos campos de título ou resumo ou palavras-chave. Essa busca no sítio eletrônico perfez um total de 587 artigos selecionados. Subsequentemente, houve o refinamento da amostra pela análise dos títulos dos artigos, eliminando os de pouca aderência ao tema. Assim, nessa etapa, foram selecionados 201 artigos. Posteriormente, desse total, foi realizada a leitura prévia dos resumos, o que resultou em 51 artigos. Na sequência, o crivo foi a leitura do artigo completo, como forma de identificação de apenas bibliometrias e, destas, apenas as da área selecionada. Dessa forma, obteve-se 37 artigos selecionados, os quais compuseram o banco de dados desta pesquisa.

Para a geração das análises com uma maior quantidade de detalhes, a coleta de dados foi desmembrada em dois blocos. O primeiro evidencia as informações dos dados dos 37 artigos selecionados e o segundo resume as informações dos artigos tratados no primeiro.

Como forma de resumir parte da metodologia, a Figura 1 ilustra algumas das etapas perpassadas para concretização dessa pesquisa.

\section{[Figura 1 - Localizada após as Referências]}

Como forma de exposição e análise dos dados coletados, esses foram descritos e apresentados no formato de figuras. Ademais, duas outras técnicas foram utilizadas com o auxílio do software Iramuteq 0.7 alpha 2. Na primeira, construíram-se sociometrias (nuvem de palavras - Word Clouds), com base nas palavras-chave e nos resumos dos artigos do primeiro bloco, objetivando mostrar a frequência com que apareceram no texto. Essa técnica possibilitou a visualização lexicográfica das palavras contidas no corpus textual examinado. Vale ressaltar que foi utilizado um corpus específico das palavras-chave e dos resumos com o intuito de comparação entre as sociometrias. 
A segunda etapa analisou a Classificação Hierárquica Decrescente (CHD) e a Análise Fatorial de Correspondência (AFC) para verificação dos agrupamentos dos resumos dos artigos estudados. A CHD foi representada por meio de um dendograma a fim de demonstrar como as palavras, textos ou expressões se distribuem entre classes. A referida análise permitiu observar, estatisticamente, como as palavras do corpus se assemelham ou se distinguem entre si. A AFC foi realizada com o propósito de representar as palavras apresentadas no CHD, que são as mais adequadas ao corpus, em uma distribuição no plano cartesiano. Essa representação descreveu as classes em formato de clusters. $\mathrm{O}$ objetivo da subdivisão em classes correlacionadas parte do princípio de que os vocábulos estejam distribuídos em grupos (KRONBERGER; WAGNER, 2002).

\section{RESULTADOS}

Esta seção abordará, em dois blocos, os resultados obtidos na pesquisa. O primeiro bloco representa os dados dos artigos bibliométricos que compuseram a amostra deste estudo. O segundo refere-se aos dados inclusos nos artigos trabalhados nas bibliometrias analisadas, ou seja, os dados dos artigos do primeiro bloco. Essa distinção foi necessária por esta pesquisa, de forma inovadora, para realizar uma bibliometria de bibliometrias.

\section{BLOCO 1: DADOS DOS ARTIGOS DA AMOSTRA (BIBLIOMETRIAS)}

As características dos artigos bibliométricos na área de marketing, que compuseram a porção final do estudo, foram oriundos da base de busca Spell, sendo consideradas 37 bibliometrias. O ano de 2015 abarcou o maior número de bibliometrias publicadas na área, 18,9\% do total. Por meio da Figura 2, percebeu-se uma elevação na quantidade a partir do ano de 2012 (10,8\%); 2013, 2014 e 2016 alcançaram o mesmo percentual (13,5\%). Em comparação aos anos de maior publicação, 2017 possui 16,2\% dos artigos.

No que tange aos periódicos científicos nos quais as bibliometrias foram publicadas, os artigos foram distribuídos em 28 revistas. A 'Revista de Ciências da Administração (RCA)' e a 'Revista Brasileira de Marketing (ReMark)' foram as que tiveram uma predominância de publicações (10,8\%), seguidas por outras três: 'Revista Pretexto'; 'Revista de Administração da UNIMEP (RAU)' e 'Revista de Administração Contemporânea (RAC)', cada uma com $5,4 \%$. 
Os demais periódicos foram responsáveis por uma publicação cada: 'Perspectivas em Gestão \& Conhecimento (PG\&C)', 'Revista Adm. MADE'; 'Revista Administração em Diálogo (RAD)'; 'Revista Brasileira de Estratégia (REBRAE)'; 'Revista Brasileira de Pesquisa em Turismo (RBTUR)'; 'Revista da Micro e Pequena Empresa (FACCAMP)'; 'Revista de Administração da UFSM (ReAUFSM)'; 'Revista de Administração de Empresas (RAE)'; 'Revista de Administração e Contabilidade da Unisinos (BASE)'; 'Revista de Administração FACES Journal'; 'Revista de Administração Mackenzie (RAM)'; 'Revista de Administração, Contabilidade e Economia da FUNDACE (RACEF)'; 'Revista de Administração, Contabilidade e Sustentabilidade (REUNIR)'; 'Revista de Gestão Social e Ambiental (RGSA)'; 'Revista Eletrônica de Administração (REAd)'; 'Revista Eletrônica Gestão e Serviços (REGS)'; 'Revista Gestão \& Tecnologia (G\&T)'; 'Revista Ibero-Americana de Estratégia (RIAE)'; 'Revista Organizações em contexto (ROC)'; 'Revista Pensamento Contemporâneo em Administração (RPCA)'; 'Revista Turismo - Visão e Ação; Sociedade, Contabilidade e Gestão (SCG)' e 'Teoria e Prática em Administração (TPA)'.

Conforme classificação do Qualis/Capes (Coordenação de Aperfeiçoamento de Pessoal de Nível Superior) referente ao quadriênio 2013-2016, observou-se que 15 das 28 revistas têm classificação B2 (40,5\%), acompanhada da classificação B1, com 14 artigos (37,8\%). Ainda se depreende que a classificação A2, maior Qualis nacional em Administração, esteve presente em apenas 3 bibliometrias $(8,1 \%)$.

Entre as bibliometrias analisadas, ressalta-se que, em sua maioria, a fonte de dados mais utilizada foi oriunda do evento Encontro da Associação Nacional de Pós-Graduação e Pesquisa em Administração (EnANPAD), (31,2\%). Ademais, foram expressivas as publicações com bases provenientes de periódicos nacionais (24,1\%), Encontro de Marketing da ANPAD - EMA (22,2\%), seguido de 14,8\% de periódicos internacionais. Como exemplos de outras fontes de dados empregadas pode-se ressaltar: ANPAD; banco de teses da Capes; Biblioteca Virtual em Saúde (BVS); EBSCO; 3ES; Semead; Spell; Scielo; Scopus; Web of Knowledge e Web of Science. Salienta-se que a origem dos dados para a construção das bibliometrias podem ter tido mais de uma fonte.

Outrossim, a análise das bibliometrias possibilitou identificar os autores e as instituições com maior número de artigos. As instituições que prevaleceram em quantidade de publicações foram: Universidade Federal do Rio Grande do Sul (UFRGS) com 6 artigos; Universidade Nove de Julho (UNINOVE) e a Universidade de São Paulo (USP), ambas com 4 publicações. 
Os autores Irene Raguenet Troccoli e Carlos Alberto Vargas Rossi se destacaram, ao publicarem no período, 4 e 3 artigos respectivamente.

Em um total de 37 bibliometrias, foram constatados 106 autores, perfazendo uma média de 2,86 autores por artigo, pertencentes a 35 instituições. Ademais, a agenda de pesquisa e as limitações dos estudos foram discutidas em $81,08 \%$ e 64,86\% dos artigos respectivamente.

Os softwares utilizados nas bibliometrias foram expostos em 48,65\% dos artigos. O Microsoft Excel, com 29,73\%, e o Statistical Package for Social Sciences (SPSS), com 21,62\%, foram os softwares mais utilizados pelos autores. Demais softwares também foram identificados no processo de análise dos dados: Google Docs; VosViewer; Proknow-C; Endnote X3; Google Scholar Citations; BibExcel; NodeXL do Excel; algoritmos Harel-Koren Fast Multiscale e Fruchterman-Reingold; Wordlle e Ucinet.

\section{BLOCO 2: APRENTAÇÃO DOS DADOS CONTIDOS NOS ARTIGOS TRABALHADOS NAS BIBLIOMETRIAS DO BLOCO 1}

A análise dessa seção contempla 15781 artigos estudados no conjunto das bibliometrias descritas no Bloco1, perfazendo uma média de 563,61 artigos por publicação. Vale ressaltar que, em relação a essa quantidade, o valor mínimo foi de 7 artigos por bibliometria e o valor máximo de 5270 artigos por bibliometria (desvio padrão $=1049,54)$. O intervalo de maior frequência foi o de 01 a 99 artigos (56,8\%), seguido de 13,5\% que analisaram entre 100 e 199 artigos. A lacuna composta por bibliometrias com mais de 1000 artigos $(10,8 \%)$ diversifica a proporção do tamanho das amostras.

A grande maioria das bibliometrias representou suas análises utilizando tabelas $(91,9 \%)$; seguido de gráficos $(54,1 \%)$; redes $(16,2 \%)$ e imagens $(10,8 \%)$. A utilização de quadros e nuvens de palavras (Word Clouds) pôde ser observada em 2,7\% cada. Contudo, deve ser ressaltado que alguns artigos utilizaram mais de um modo de representação dos dados.

Foi possível observar que a quantidade de artigos por ano obteve $64,9 \%$ de apontamentos, as bases de dados ou periódicos foram informadas em 54,1\%, e a quantidade por instituição em $32,4 \%$. No que tange ao número de autores, pode ser destacado que $32,4 \%$ dos artigos apresentaram a quantidade de autores por publicação e a quantidade total, $24,3 \%$ apresentaram a quantidade de autores por artigo e $21,6 \%$ a instituição. 
Relativo ao item 'referências', 35,1\% informaram o nome dos autores mais citados. Já $5,4 \%$ tiveram as referências informadas no texto e também destacaram a quantidade total. $\mathrm{O}$ item 'metodologia' retrata que o método de pesquisa foi exposto em $51,4 \%$ dos artigos, a fonte de dados foi evidenciada em $10,8 \%$, o objetivo dos estudos em $8,1 \%$ e o modelo de coleta de dados em $5,4 \%$.

No que tange à 'metodologia', pode ser destacado que 35,14\% dos artigos apresentaram o enquadramento do estudo, e destes, $81,34 \%$ foram teórico-empíricos e 18,66\% ensaios teóricos.

A análise metodológica analisada foi: instrumento de coleta de dados (41\%); abordagem metodológica e natureza da pesquisa (38\%); tipo de pesquisa (35\%); técnica de análise (22\%); fonte de dados $(11 \%)$ e tipo de amostra $(5 \%)$. O survey $(35,98 \%)$ e a entrevista semiestruturada $(23,44 \%)$ foram os principais instrumentos de coleta de dados. Na abordagem metodológica, foram mais expressivos o estudo de caso $(9,76 \%)$ e a análise documental $(6,74 \%)$. Os principais tipos de pesquisa foram a pesquisa exploratória $(28,76 \%)$ e a descritiva $(25,51)$. Em relação à técnica de análise de dados, 46,41\% utilizaram a análise multivariada e 17,33\% a estatística descritiva.

Quanto à natureza, foram observadas as pesquisas quantitativas $(5,43 \%)$; qualitativas $(3,6 \%)$ e quanti-qualitativas $(0,61 \%)$. Como fonte de dados das pesquisas, prevaleceram as do tipo primário, com $61,48 \%$, e, no que diz respeito ao tipo de amostra, $15 \%$ das informadas apontaram ser não-probabilísticas e 5,08\% probabilísticas.

\section{DISCUSSÃO E AGENDA DE PESQUISA}

O campo de marketing, considerado como a grande área nesta pesquisa, gerou reflexões sobre a relevância da temática, possibilitando sugestões para futuros estudos. Nesse sentido, as primeiras discussões irão partir das análises das temáticas já estudadas nos artigos pesquisados.

$\mathrm{O}$ ‘consumo' foi a temática mais trabalhada (11\%) nas bibliometrias pesquisadas, tendo seu panorama de bibliometria entre os anos de 1997 e 2013. As bibliometrias, além de tratarem esse assunto de forma isolada, também o relacionaram com a cultura e com o saciamento/atenção. Na perspectiva de Bauman (2008), no início do século XX, o consumo era ligado à durabilidade e à segurança, no entanto, para o consumidor nos dias atuais, a rápida substituição dos produtos é que traz prazer e felicidade. A "homogeneização generalizada de gostos e preferências" tem sido utilizada por Levitt (1990, p.49) como forma de expressar que 
o consumo não está acontecendo isoladamente, mas sim como consequência de um mercado mais veloz e, para ele, sem pátria.

Também chamam a atenção as temáticas 'marketing' e 'estratégia', que estiveram presentes em $8 \%$ dos artigos. O marketing, na visão de Faria (2006), tem perdido sua identidade no Brasil. Vieira (2000) complementa que essa perda pode ser devido às publicações da área estarem concentradas em torno do tema 'comportamento do consumidor' e os artigos terem, em sua maioria, abordagens quantitativas como forma de fazer com que o marketing seja legitimado como ciência.

Já a temática 'estratégia' está altamente entrelaçada à temática do 'marketing' (WILLIAMSON, 2010; THEODOSIOU; KEHAGIAS; KATSIKEA, 2012; BARNEY, 2013). Porter (1980) já revelava essa relação e apontava que a estratégia de marketing e o planejamento estratégico devem estar alinhados para que o desempenho organizacional seja alavancado pelas vantagens competitivas.

Cabe considerar que algumas bibliometrias foram enfáticas em considerar a sua coleta de dados em artigos que relacionavam o marketing com aspectos metodológicos e fenomenológicos, como: estudo de caso; análise discriminante; etnografia; epistemologia e survey. No tocante às ciências, dois artigos abordaram a relação do marketing com as ciências sociais e com as outras ciências. Nesse sentido, o marketing, sendo analisado em artigos relacionados a uma técnica específica de metodologia, fenomenologia, ou relacionado a outras ciências, poderia compor uma agenda de pesquisa para futuros trabalhos fazendo uso, por exemplo, de modelagens de equações estruturais (MEE), citadas por Vieira (2000), ou relacionando o marketing às séries temporais.

Outras temáticas tratadas em 5\% das bibliometrias foram: Comportamento do consumidor (CO); marketing de relacionamento (CRM) e marketing de serviços. Os demais assuntos foram estudados em uma bibliometria, a saber: marketing ambiental; análise discriminante; B2B (Business to Business); brand equity; ciências sociais; estudos críticos em administração (CMS); comércio justo; consumidor no varejo; epistemologia; estudo de caso; imagem; inovação em serviços; lealdade; etnografia; pequenas empresas; qualidade em serviços; responsabilidade socioambiental empresarial (RSAE); segmentação de mercado; marketing social e survey.

Do ponto de vista da sociometria (nuvem de palavras - Word Cloud), construída a partir das palavras-chave e dos resumos das bibliometrias (Figuras 2 e 3), algumas reflexões também podem ser discutidas. Das 96 palavras-chave encontradas nos artigos, 14 termos relacionaram- 
se ao marketing, o que já era de se esperar por se tratarem de bibliometrias relacionadas a essa área. No entanto, em comparação com a nuvem de palavras produzida a partir dos resumos, o mesmo não aconteceu, e os termos 'artigo', 'estudo' e 'pesquisa' foram os mais abordados nos textos, ficando o marketing no quarto lugar. Vale ressaltar que o termo ‘bibliométrico' esteve evidente nas duas nuvens representadas.

\section{[Figura 2 - Localizada após as Referências]}

\section{[Figura 3 - Localizada após as Referências]}

Outras discussões puderam ser realizadas por meio da análise textual dos resumos apresentados nas bibliometrias de marketing incorporadas neste estudo. O corpus geral foi composto por 37 textos (resumos dos artigos), distribuídos em 173 seguimentos de texto (ST), com aproveitamento de $100 \%$. Assomaram a ocorrência de 6131 palavras, sendo que 1242 são distintas. Foram observadas as palavras com frequência superior ou igual a três ocorrências e aplicadas nas análises de Classificação Hierárquica Decrescente (CHD) e Análise Fatorial de Correspondência (AFC).

A análise CHD possibilitou a identificação dos textos correlacionados no corpus textual analisado, formando um esquema hierárquico das classes definidas no estudo. Compareceram na análise três classes relevantes para categorização. São elas: Classe1, contemplando 51,5\% de seguimentos textuais; Classe 2, com 38,1\%; e Classe 3, com 10,4\%. Os detalhes dessa distribuição podem ser observados na Figura 4. A subdivisão das classes correlacionadas nos vocábulos textuais faz com que haja uma distribuição grupal dos trechos de modo adequado (KRONBERGER; WAGNER, 2002).

\section{[Figura 4 - Localizada após as Referências]}

A Figura 5 retrata a AFC, com base nas frequências e valores de correlação dos Quiquadrados $\left(\mathrm{X}^{2}\right)$. Nela, é possível observar como as palavras de cada classe estão distribuídas no plano fatorial. Para essa análise, foram considerados os segmentos de variáveis ativas com $\mathrm{X}^{2}$ $\geq 3,86(\mathrm{p}<0,05)$. Cabe salientar que alguns termos não foram demonstrados no dendograma por não apresentarem grau de significância estatística. 
Cada agrupamento representado no plano fatorial da Figura 5 refere-se a uma classe hierárquica decrescente. A Classe 1 está representada pelo círculo numerado em 1, assim como a Classe 2 pelo círculo 2 e a Classe 3 pelo círculo 3 .

\section{[Figura 5 - Localizada após as Referências]}

Cabe salientar que as três classes estão distribuídas em duas ramificações (A e B), denominadas como subcorpus do corpus geral indicado na análise. O primeiro subcorpus (A) 'consumidor' refere-se à Classe 3, onde são apresentadas as palavras relacionadas ao alvo principal do marketing, o consumidor, o consumo e em geral ao saciamento, compra e atenção que devem ser objetivos do mercado. O segundo subcorpus incorpora as Classes 1 e 2. A Classe 1 'estudo bibliométrico', contempla as palavras 'bibliométrico', 'EnANPAD' e 'EMA', constituindo as bases e termos introdutórios das bibliometrias estudadas. Em relação à Classe 2 'objetivos da pesquisa', tem-se que as palavras 'pesquisas futuras', 'agenda' e 'limitações' remetem ao nome da classe.

A Figura 5 permitiu, ainda, observar que a posição da Classe 2 no plano cartesiano seria um elo de ligação entre as Classes 1 e 3 . Os objetivos da pesquisa - Classe 2 - foram alinhados com as palavras-chave pesquisadas no início deste estudo, que estão contempladas no estudo bibliométrico - Classe 1 - em junção com o consumidor - Classe 3.

\section{CONSIDERAÇÕES FINAIS}

Este artigo, de maneira inovadora realizou uma bibliometria de bibliometrias, e pretendeu apresentar um panorama da área de marketing, retratando um condensado desses trabalhos e dos artigos trabalhados de modo a resumir para pesquisadores e leitores as produções científicas que têm como pressuposto essa abordagem. Nesse sentido, foram identificadas as variáveis coletadas nos estudos, e foi possível sugerir uma agenda de pesquisa.

Constatou-se a predominância das revistas 'Revista de Ciências da Administração (RCA)' e a 'Revista Brasileira de Marketing (ReMark)' com a maioria de artigos dessa natureza. Em relação aos artigos contidos nas publicações, verificou-se que os estudos de caráter teórico-empíricos e de natureza quantitativa ocorreram em maior número. Entre esses 
estudos, também se destacaram aqueles escritos por mais de um autor. Quanto às categorias mais tratadas pelos autores, as temáticas dos artigos e a quantidade deles por ano prevaleceram sobre as demais.

Apesar das contribuições, algumas limitações podem ser apontadas, como a escolha de uma base de dados específica, embora o objetivo deste estudo não fosse preencher todas as lacunas da literatura nacional.

Por fim, espera-se que este estudo possa ter gerado apontamentos e resultados que forneçam subsídios para pesquisadores sobre os aspectos que estão sendo tratados ao se publicar uma bibliometria, mormente na área de marketing. Aspectos específicos da área de marketing também podem auxiliar escritores, visto que foram observadas as temáticas que já foram ou que poderiam ser pesquisadas. Assim, as possibilidades apresentadas são múltiplas, visto que vários assuntos ainda não foram tratados com a mesma metodologia utilizada neste.

\section{AGRADECIMENTOS}

Agradecemos o apoio financeiro da Coordenação de Aperfeiçoamento de Pessoal de Nível Superior (CAPES) e da Fundação de Amparo à Pesquisa do Estado de Minas Gerais (FAPEMIG).

\section{CONFLITOS DE INTERESSE}

Nada a declarar.

\section{REFERÊNCIAS}

ANDERSON, P. F. Marketing, Scientific Progress, and Scientific Method. Journal of Marketing, v. 47, n. 4, 1983, p. 18-31.

ARAÚJO, C. A. A. Bibliometria: evolução história e questões atuais. Revista em Questão, v. 12, n. 1, 2006, p.11-32.

BAKER, M. J. Marketing - philosophy or function? Marketing theory: a student text. New York: Thomson Learning, 2000. 
BAKIR, A; VITELL, S. J.; ROSE, G. M. Publications in major Marketing journals: an analyis of scholars and Marketing departments. Journal of Marketing Education, v. 22, n. 2, 2000, p. 99-107.

BARTELS, R. The History of Marketing Thought. Columbus: Publishing Horizons, 1988.

BAUMAN, Z. Vida para o Consumo: a transformação das pessoas em mercadorias. Rio de Janeiro: Jorge Zahar, 2008.

BREI, V. A.; LIBERALI, G. O uso de modelagem em equações estruturais na área de Marketing do Brasil. In: Encontro Nacional da Associação Nacional dos Programas de PósGraduação em Administração (ANPAD), 28, 2004, Porto Alegre. Anais.

COTE, J. A; LEONG, S. M.; COTE, J. Assessing the influence of Journal of consumer Research: a citation analysis. Journal of Consumer Research, v. 18, n. 3, 1991, p. 402-411.

FARIA, A. Em busca de uma agenda brasileira de pesquisa em estratégia de marketing. Revista de Administração de Empresas - RAE, v. 46, n. 4, 2006, p. 13-24.

GUEDES, V. L. S.; BORSCHIVER, S. Bibliometria: uma ferramenta estatística para a gestão da informação e do conhecimento, em sistemas de informação, de comunicação e de avaliação científica e tecnológica. Encontro Nacional de Ciência da Informação, v. 6, p. 1-18, 2005. Anais.

HULME, E. W. Statistical bibliography in relation to the growth of modern civilization. London: Grafton, 1923.

HUNT, S. D. Foundations of marketing theory: toward a general theory of marketing. Armonk, NY: ME Sharpe, 2002.

HUNT, S. General Theories and the Fundamental Explananda of Marketing. Journal of Marketing, v. 47, n. 4, 1983, p. 9-17. 
KERIN, R. A. In pursuit of an ideal: the editorial and literary history of the Journal of Marketing. Journal of Marketing, v. 60, n. 1, 1996, p. 1-13.

KRONBERGER, N. E.; WAGNER, W. Palavras-chave em contexto: análise estatística de textos. In: BAUER, M. W.; GASKELL, G. (Orgs.). Pesquisa qualitativa com texto, imagem e som: um manual prático. Petrópolis: Vozes, 2002.

LEONE, R. P; ROBINSON, L. M; BRAGGE, J.; SOMERVUORI, O. A citation and profiling analysis of pricing research from 1980 to 2010. Journal of Business Research, v. 65, n. 7, 2011, p. $1010-1024$.

LEONG, S. M. A citation analysis of the Journal of Consumer Research. Journal of Consumer Research, v. 15, n. 4, 1989, p. 492-497.

LEVITT, T. A. Imaginação de Marketing. 2. ed. São Paulo: Atlas, 1990.

MACHADO-DA-SILVA, C. L.; CUNHA, V. C.; \& AMBONI, N. Organizações: o estado da arte da produção acadêmica no Brasil. In: Encontro Anual da Associação Nacional Dos Programas De Pós-Graduação Em Administração (ANPAD), 14, 1990, Florianópolis. Anais.

PERIN, M. G; SAMPAIO, C. H; FROEMMING, L. M. S; LUCE, F. B. A pesquisa survey em artigos de Marketing nos Enanpads da década de 90. In: Encontro Nacional da Associação Nacional dos Programas de Pós-Graduação em Administração (ANPAD), 24, 2000, Florianópolis. Anais.

PRITCHARD, A. Statistical bibliography or bibliometrics? Journal of Documentation, v. 25, n. 4, 1969, p. 348-349.

RAMOS-RODRÍGUEZ, A.; RUÍZ-NAVARRO, J. Changes in the Intellectual Structure of Strategic Management Research: A Bibliometric Study of the Strategic Management Journal, 1980-2000. Strategic Management Journal, v. 25, n. 10, 2004, p. 981-1004. 
TALUKDAR, D; HARIHARAN, V. G.; BOO, C. Empirical regularity in academic research productivity patterns in Marketing. International Journal of Research in Marketing, v. 28, n. 3, 2011, p. 248-257.

TELLIS, G. J; CHANDY, R. K.; ACKERMAN, D. S. Search of diversity: the record of major Marketing journals. Journal of Marketing Research, v. 36, n. 1, 1999, p. 120-131.

VANTI, N. Da bibliometria à webometria: uma exploração conceitual dos mecanismos utilizados para medir o registro da informação e a difusão do conhecimento. Ciência da Informação, v. 31, n. 2, 2002, p. 152-162.

VIEIRA, F. G. D. Narciso sem espelho: a publicação brasileira de marketing. Revista de Administração de Empresas - RAE, v. 43, n. 1, 2003, jan./fev./mar, p. 81-90.

VIEIRA, R. S. G. et al. O conhecimento de marketing sob os olhos da teoria crítica. In: ENANPAD, 16., 2002, Salvador. Anais eletrônicos. Salvador: ANPAD.

VIEIRA, F. G. D. Panorama acadêmico-científico e temáticas de estudos de marketing no Brasil. In: 24 ENANPAD, 24., 2000, Foz do Iguaçu. Anais eletrônicos. Florianópolis: ANPAD.

VIEIRA, F. G. D. Ações empresariais e prioridades de pesquisa em marketing: tendências no Brasil e no mundo segundo a percepção dos acadêmicos brasileiros. In: ENANPAD, 23., 1999, Foz do Iguaçu. Anais eletrônicos. Foz do Iguaçu: ANPAD.

VIEIRA, F. G. D. Por quem os sinos dobram? Uma análise da publicação científica na área de marketing do ENANPAD. In: ENANPAD, 22., 1998, Foz do Iguaçu. Anais eletrônicos. Foz do Iguaçu: ANPAD. 


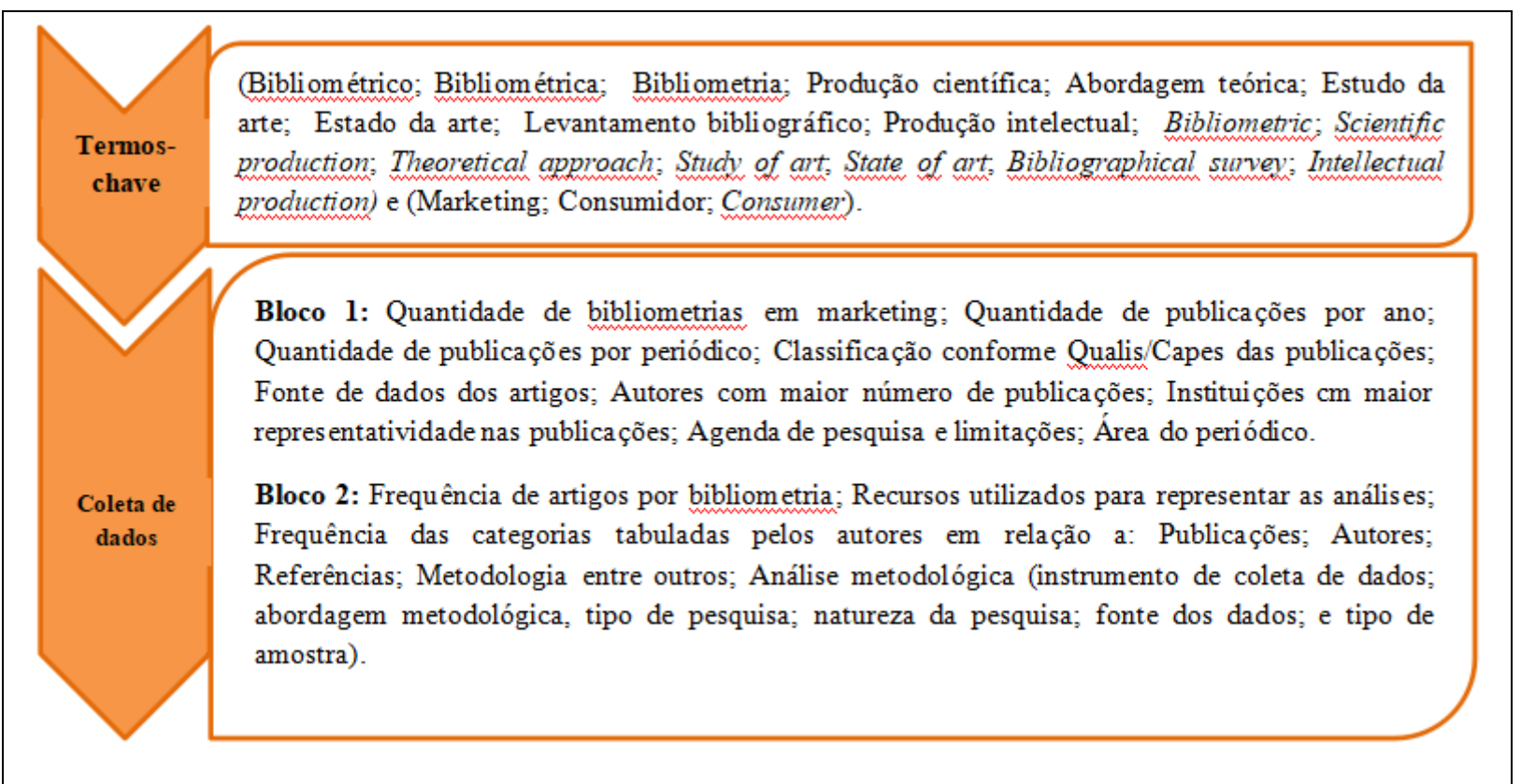

Figura 1: Etapas do método de pesquisa.

Fonte: Elaborada pelos autores (2019). 


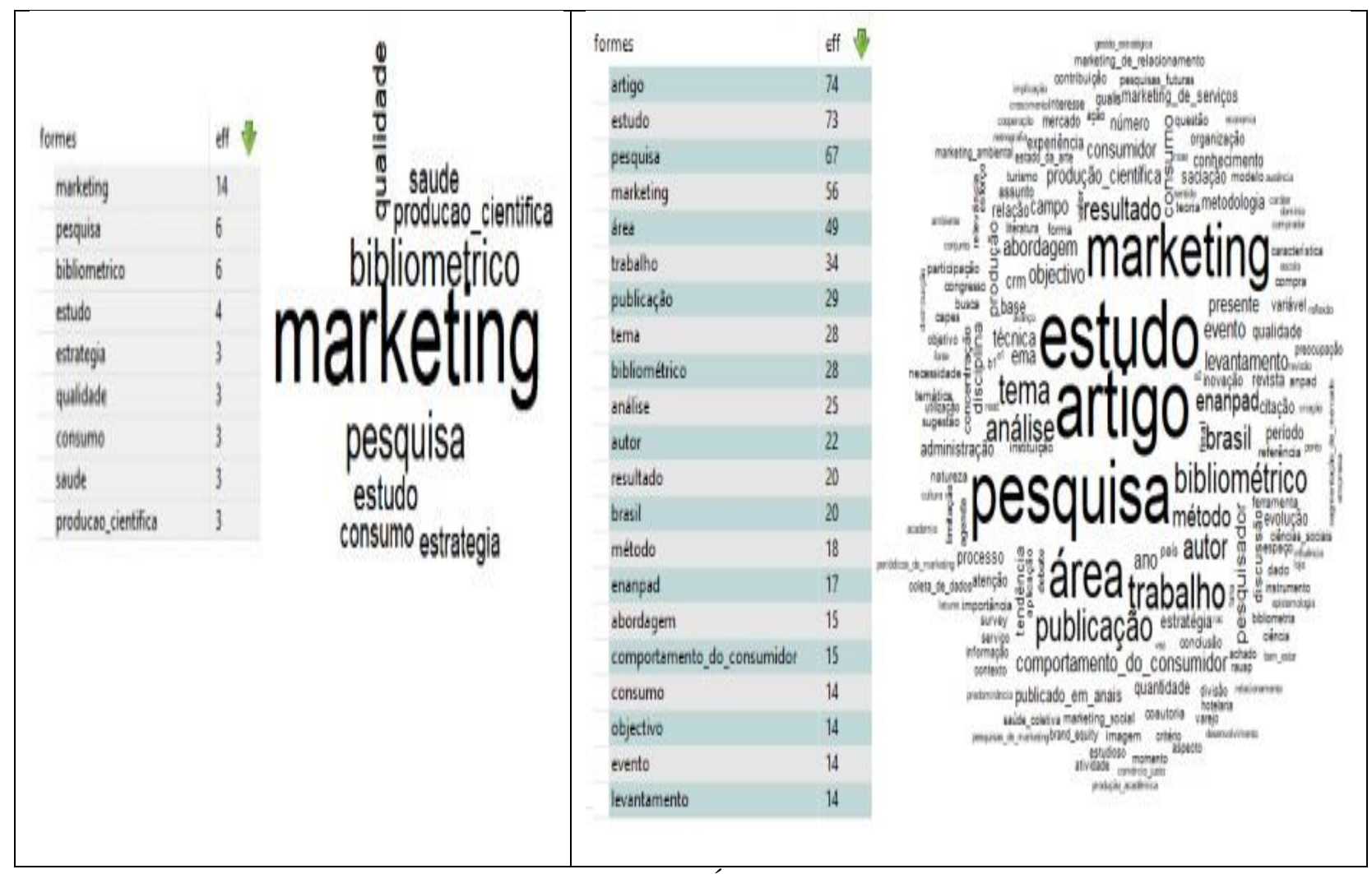

Figura 2: Nuvem de Palavras-Chave

Fonte: Elaborada pelos autores (2019).
Figura 3: Árvore de Palavras-Chave

Fonte: Elaborada pelos autores (2019).

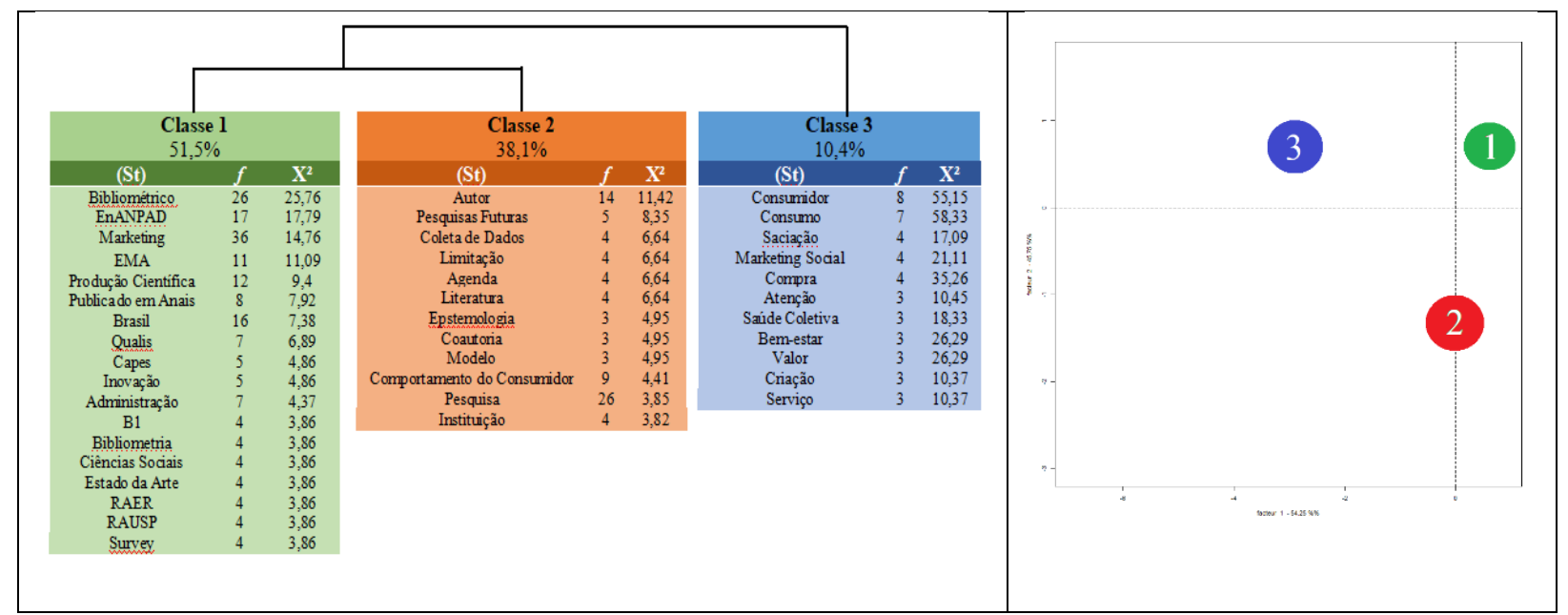

Figura 4: Dendograma CHD

Fonte: Elaborada pelos autores (2019).
Figura 5: Orientação AFC

Fonte: Elaborada pelos autores (2019). 